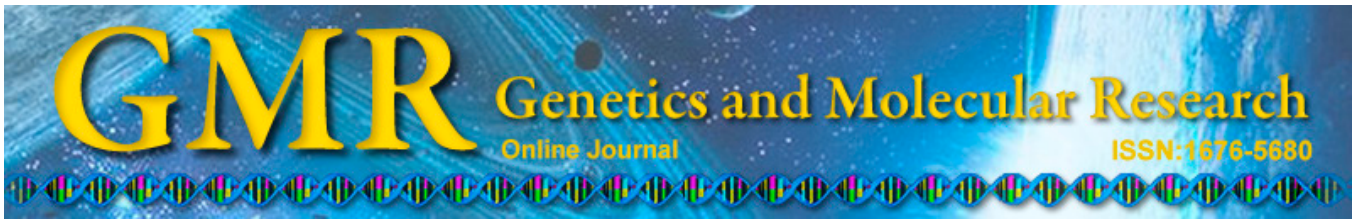

\title{
Effects of acrylonitrile on lymphocyte lipid rafts and RAS/RAF/MAPK/ERK signaling pathways
}

\author{
X.J. Li, B. Li*, J.S. Huang, J.M. Shi, P. Wang, W. Fan and Y.L. Zhou* \\ Department of Occupational Health, Jinshan Hospital, Fudan University, \\ Shanghai, China \\ *These authors contributed equally to this study. \\ Corresponding authors: Y.L. Zhou / B. Li \\ E-mail: zhouy12000@vip.sina.com / libing22@126.com
}

Genet. Mol. Res. 13 (3): 7747-7756 (2014)

Received May 7, 2014

Accepted September 11, 2014

Published September 26, 2014

DOI http://dx.doi.org/10.4238/2014.September.26.12

\begin{abstract}
Acrylonitrile (ACN) is a widely used chemical in the production of plastics, resins, nitriles, acrylic fibers, and synthetic rubber. Previous epidemiological investigations and animal studies have confirmed that ACN affects the lymphocytes and spleen. However, the immune toxicity mechanism is unknown. Lipid rafts are cell membrane structures that are rich in cholesterol and involved in cell signal transduction. The B cell lymophoma-10 (Bc110) protein is a joint protein that is important in lymphocyte development and signal pathways. This study was conducted to examine the in vitro effects of ACN. We separated lipid rafts, and analyzed Bcl10 protein and caveolin. Western blotting was used to detect mitogen-activated protein kinase (MAPK) and phosphorylated MAPK levels. The results indicated that with increasing $\mathrm{ACN}$ concentration, the total amount of Bcl10 remained stable, but was concentrated mainly in part 4 to part 11 in electrophoretic band district which is high density in gradient centrifugation. Caveolin-1 was evaluated as a lipid raft marker protein; caveolin-1 content and position were relatively unchanged. Western blotting showed that in a certain range, MAPK protein was secreted at
\end{abstract}


a higher level. At some ACN exposure levels, MAPK protein secretion was significantly decreased compared to the control group $(\mathrm{P}<0.05)$. These results indicate that $\mathrm{ACN}$ can cause immune toxicity by damaging lipid raft structures, causing Bcl10 protein and lipid raft separation and restraining Ras-Raf-MAPK-extracellular signal-regulated kinase signaling pathways.

Key words: Acrylonitrile; Bcl10 protein; Caveolin-1; Lipid rafts; MAPK

\section{INTRODUCTION}

Acrylonitrile $(\mathrm{ACN})$ is a volatile, toxic liquid monomer that is widely used in the manufacture of synthetic rubber, styrene plastics, and acrylic fibers, among other products. ACN production and demand has increased since 1979, particularly in the Asia-Pacific region (Yuanqing et al., 2013). ACN production of Shanghai accounts for nearly $50 \%$ of the national output and thousands of people are exposed to ACN in production.

Various long-term ACN toxicity studies examining its neurotoxicity, endocrine toxicity, reproductive toxicity, and aging have been conducted. Previous epidemiological investigations of ACN immunotoxicity and animal studies have confirmed that ACN affects the lymphocytes and spleen. However, the mechanism of immunotoxicity remains unclear. Based on previous studies examining immune synapse formation, $\mathrm{T}$ cell receptor-mediated signal transduction studies could reveal whether lipid rafts and joint B cell lymophoma-10 (Bcl10) protein play important roles in signal transduction pathways.

Lipid rafts, which are present in all leukocyte cells, are currently defined as dynamicordered nanoscale assemblies of proteins and lipids of the plasma membrane and other intracellular membranes, such as Golgi membranes, that associate and dissociate on a sub-second timescale (Hancock, 2006; Pike, 2006; Simons and Gerl, 2010). They contain high levels of cholesterol, sphingolipids (such as sphingomyelin), and gangliosides. Lipid rafts are highly organized and likely exist in a liquid-ordered phase, which differs from the rest of the plasma membrane that consists mainly of phospholipids (with unsaturated tails) in a liquid-disordered phase (Simons and Vaz, 2004). They can also form stable platforms that are important in signaling pathways, such as cell signal transduction across the membrane, cell adhesion, and microbial infection. In particular, T cell-mediated signal transduction occurs in microareas. Immune synapse assembly depends on the clustering of lipid rafts, which is essential for $\mathrm{T}$ cell activation.

In addition, lipid rafts are dynamic and variable; they can move laterally and regionalize cell membranes. Lipid microdomains vary in stability, size, shape, lifespan, and molecular composition (Szoor et al., 2010). Because of their different molecular compositions, studies of lipid rafts have been complicated by imprecise nomenclature. The term caveolae has been synonymous with lipid rafts for many years, and their function is likely aided by stimulationinduced association with and recruitment of various molecules with raft affinity, as well as varying degrees of raft engagement with the cytoskeleton. Previous studies have shown that in resting T lymphocytes, lipid rafts are evenly distributed in the cell membrane. When T cell antigen is stimulated, the lipid raft gathers and collects related signaling proteins. If receptor activation takes place in an ordered lipid raft, the signaling complex is protected from other 
proteins, such as membrane phosphatases localized in the disordered region of the plasma membrane that otherwise could affect the signaling process (Young et al., 2003).

The function of rafts is likely aided by stimulation-induced association and recruitment of various molecules with raft affinity, as well as varying degrees of raft engagement with the cytoskeleton (Kusumi et al., 2005). Bcl10 plays a key role in these processes by triggering T cell receptor signals and cellular activities such as the secretion of interleukin-2 (Pike, 2006; Yang et al., 2008). These processes are essential for gene activation and actin cytoskeleton rearrangement. T cells differentiate and mature, activating the downstream Ras/ Raf/mitogen-activated protein kinase (MAPK)/extracellular signal-regulated kinase (ERK) signaling pathway, and play an important role in immunotoxicity.

\section{MATERIAL AND METHODS}

\section{Cell culture and virus}

Jurkat cell lines (Shanghai Institutes for Biological Sciences, China) were incubated in RPMI-1640 medium containing 10\% newborn bovine serum with ACN (Shanghai SSS Reagent Co., Ltd., Shanghai, China) at $37^{\circ} \mathrm{C}$ and $5 \% \mathrm{CO}_{2}$. Cells were seeded on 96 -well plates, and different concentrations of drug were added at 4,12 , and $24 \mathrm{~h}$ after adding MTT reagent (20 $\mu \mathrm{L} /$ well, Promega; Madison, WI, USA); $37^{\circ} \mathrm{C}$ after 3 -h incubation, the absorbance at $\mathrm{OD}_{450}$ was detected. Then use MTT to analyze the viability of cells in vitro to further characterize differential immune sensitivities and responses.

\section{Separation and determination of cholesterol}

Cells $\left(5 \times 10^{7}\right)$ were washed twice with ice-cold phosphate-buffered saline and lysed on ice for $30 \mathrm{~min}$ in $1 \mathrm{~mL}$ TNE-buffer $(25 \mathrm{mM}$ Tris, $\mathrm{pH} 7.5,150 \mathrm{mM} \mathrm{NaCl}, 5 \mathrm{mM}$ ethylenediaminetetraacetic acid) and complete protease inhibitor cocktail with $1 \%$ Triton X-100. The cell lysates were homogenized and then centrifuged for $5 \mathrm{~min}$ at $1000 \mathrm{~g}$ and $4^{\circ} \mathrm{C}$ to remove insoluble materials. The supernatant was mixed with $1 \mathrm{~mL} 80 \%$ sucrose in TNE buffer, placed at the bottom of ultracentrifuge tubes, and overlaid with $7 \mathrm{~mL} 30 \%$ and $3 \mathrm{~mL} 5 \%$ sucrose in TNE buffer. The cell lysates were ultracentrifuged at $4^{\circ} \mathrm{C}$ for $18 \mathrm{~h}$ at $86,313 \mathrm{~g}$ in an SW41 rotor (Beckman; Brea, CA, USA). After centrifugation, twelve 1-mL fractions were collected from the top to the bottom of the tubes and analyzed immediately by immunoblotting.

During the reaction, 3 enzymes, cholesterol ester, cholesterol oxidase, and horseradish peroxidase, were used. The Amplite Cholesterol Quantitation Assay Kit (Beijing Zhonghaoshidai Co. Ltd.) was used, which is one of the most sensitive methods available for quantifying cholesterol. The amount of cholesterol is proportional to the concentration of hydrogen peroxide formed in the enzyme coupling the reaction cycle. In the presence of peroxidase, the fluorescence intensity of Amplite Red is proportional to the concentration of hydrogen peroxide that is converted to cholesterol. The results of the assay can be readily read using a fluorescence microplate reader at excitation/emission wavelengths of 540/590 nm.

Cholesterol levels were quantitated using an Amplex Red cholesterol assay kit (Molecular Probes; Eugene, OR, USA) according to manufacturer recommendations. Fluorescence was measured using TECAN Safire II (Tecan Systems Inc.; San Jose, CA, USA). A standard curve using purified cholesterol was generated for each experiment, and then cholesterol con- 
tent was detected. CD71 is non-tagged protein in rafts, which is used as an internal standard.

\section{Western blot}

Briefly, for the Western blotting protocol, proteins were transferred to an Immobilon-P membrane (Millipore, Billerica, MA, USA). Blots were blocked for $1 \mathrm{~h}$ in $5 \%$ skim milk followed by incubation with mouse anti-rat monoclonal antibody MAPK/ERK (National Institutes of Health; Bethesda, MD, USA) (1:5000) in 2\% skim milk for $1 \mathrm{~h}$ at room temperature and then antimouse horseradish peroxidase-conjugated secondary antibody (1:5000) in 5\% skim milk for $1 \mathrm{~h}$ at room temperature. Immunoreactivity was detected by chemiluminescence (Amersham Pharmacia Biotech; Amersham, UK) and exposure to X-ray film. Band intensities were assessed using Molecular Analyst (Bio-Rad; Hercules, CA, USA) and expressed as a percentage of the saline control.

\section{Statistical analysis}

All statistical analyses were performed with the SPSS 13.0 software (SPSS, Inc.; Chicago, IL, USA). For multiple comparison procedures, analysis of variance was used followed by the Student-Newman-Keuls test for post-hoc analysis. Differences were considered to be significant when $\mathrm{P}<0.05$.

\section{RESULTS}

\section{Cell viability}

Using a microscope, we observed that the cultured cells in the blank control group were close to the bottom of the bottle, which showed strong refraction. In the exposure group, the cells shrinked and floated. Viability was also assessed by determining optical density after ACN treatment, which indicates the degree of cytotoxicity induced by ACN (F $=215.21, \mathrm{P}<$ 0.05 , Figure 1).

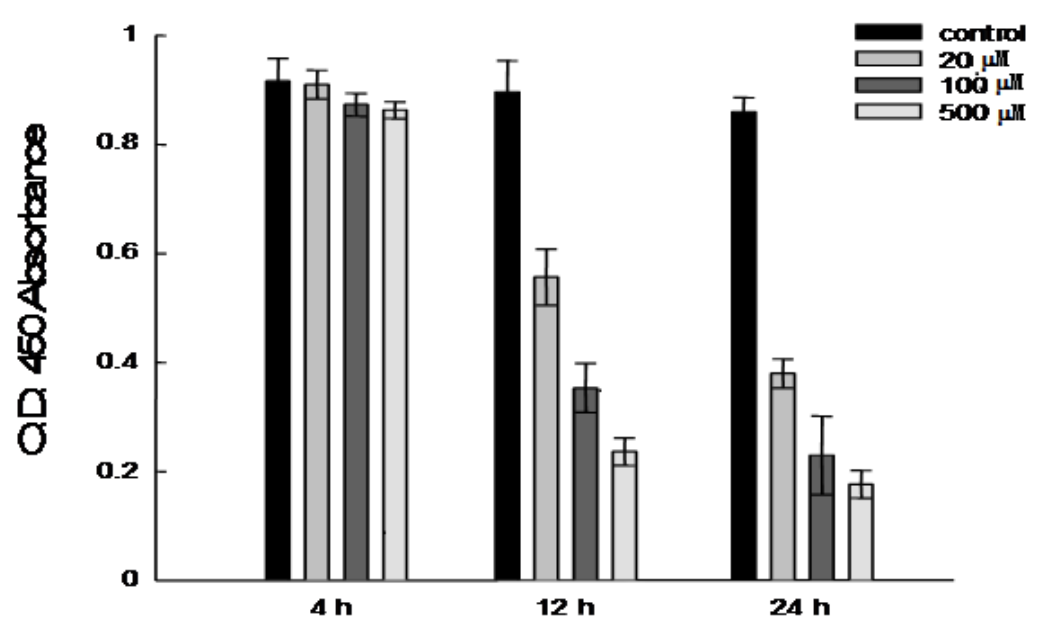

Figure 1. MTT assay with different concentrations of acrylonitrile to determine changes in cell survival. 


\section{Membrane cholesterol testing}

Cholesterol is required to build and maintain cell membranes. The results showed that at higher ACN concentration, cytotoxicity was greater but cholesterol was lower, suggesting the greater destruction degree of ACN toward lipid rafts. There were statistically significant differences between each contamination group and the control group $(\mathrm{P}<0.05)$ (Figure 2).

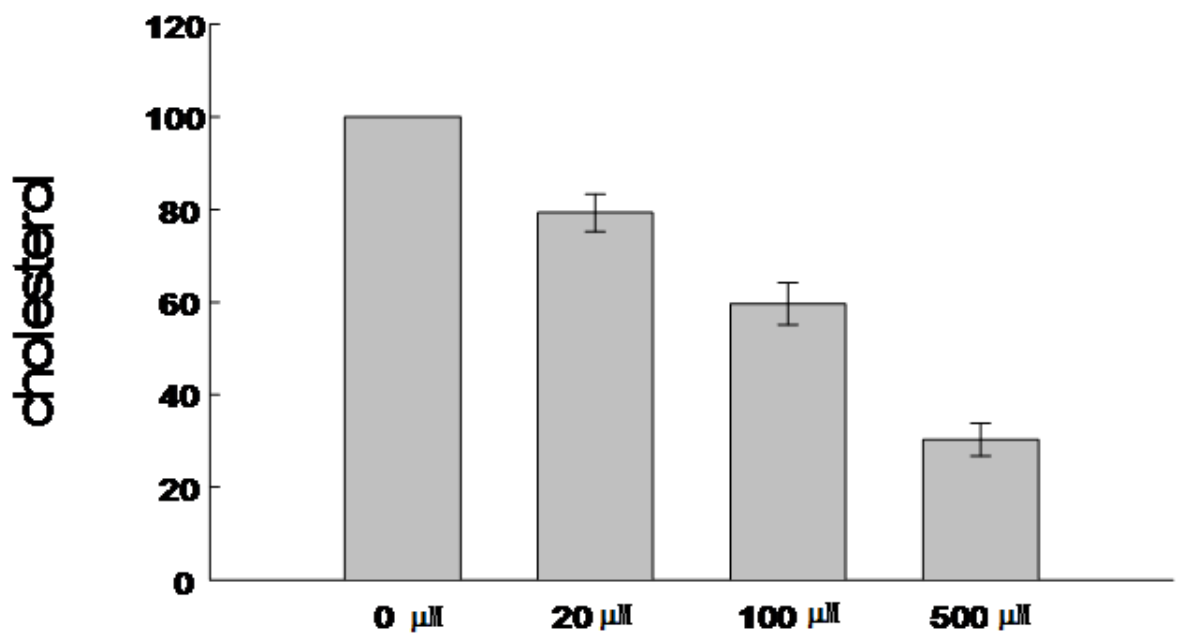

Figure 2. Effect of cholesterol after treatment with different concentrations of acrylonitrile.

\section{Bcl10 protein and caveolin-1}

$\mathrm{Bcl10}$ is a joint protein that can act during antigen receptor-mediated nuclear factor- $\mathrm{B}$ activation, lipid raft gathering, and T cell differentiation and maturation (Huang et al., 2010).

In the resting state, Bcl10 protein is evenly distributed in the Jurkat cell membrane, concentrated in lipid raft group areas 3, 4, 5, 6, 7, 9, and 10, while Bcl10 protein is redistributed to areas $8,9,10,11$, and 12 with $\mathrm{ACN}$ increasing concentration.

Pit is the most important type of lipid raft. Although several studies have increased the understanding of caveola functions, the role of caveolae in sepsis remains controversial. In our study, with increasing ACN concentration, the total amount of Bcl10 remained constant, but was concentrated mainly in the lower area. At ACN 0 and $20 \mu \mathrm{M}$ concentrations, caveolin-1 protein was located in low density gradient district (3, 4, 5, 6 band, Figure 3), while at ACN 100 and $500 \mu \mathrm{M}$ concentrations, it was located in high density $(8,9,10,11$ band, Figure 3$)$; the positioning of non-tagged protein CD71 in rafts was basically unchanged (Figure 3).

\section{Western blot}

After exposing the cells to ACN for $4 \mathrm{~h}$, protein samples were collected and analyzed for the presence of phosphorylated-MAPK (p-MAPK) and MAPK using Western blotting with $\beta$-actin as an internal reference. In Figure 4, the dark electrophoretic bands indicate higher pro- 
tein secretion, while the lighter bands represent lower protein secretion. After treatment with $\mathrm{ACN}$ for $4 \mathrm{~h}$, MAPK protein bands in the 20 and $100 \mu \mathrm{MACN}$ concentration groups were darker than those in the $500 \mu \mathrm{M}$ group. These results showed that after ACN exposure, the cellular immune system was activated and protein secretion increased at a specific ACN concentration, but cell viability and protein secretion decreased at other ACN concentrations (Figure 4).

A

$\mathbf{0} \mu \mathrm{mol}$

123456789101112
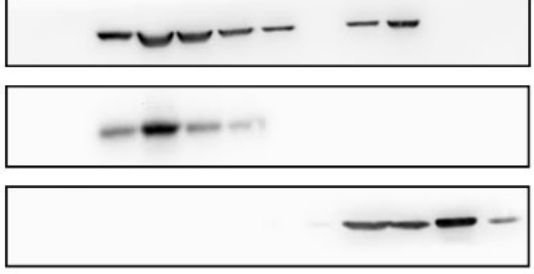

D
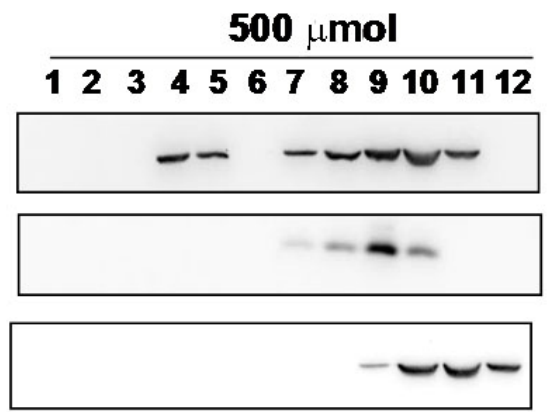

B

\section{$20 \mu \mathrm{mol}$}

1223455677899101112

Bc110

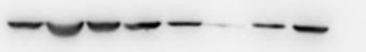

\section{Caveolin-1}

CD71

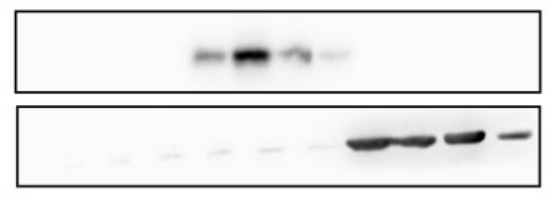

C

$100 \mu \mathrm{mol}$

Bc110

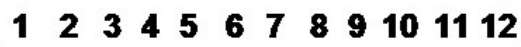

Caveolin-1

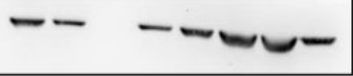

CD71
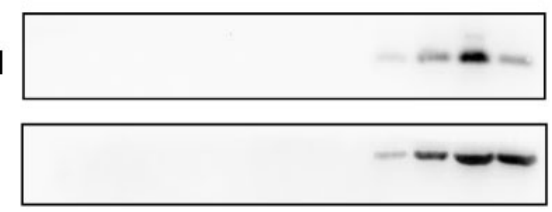

Figure 3. Jurkat cells with different concentrations of acrylonitrile on lipid rafts and lipid raft localization of Bcl10 proteins.

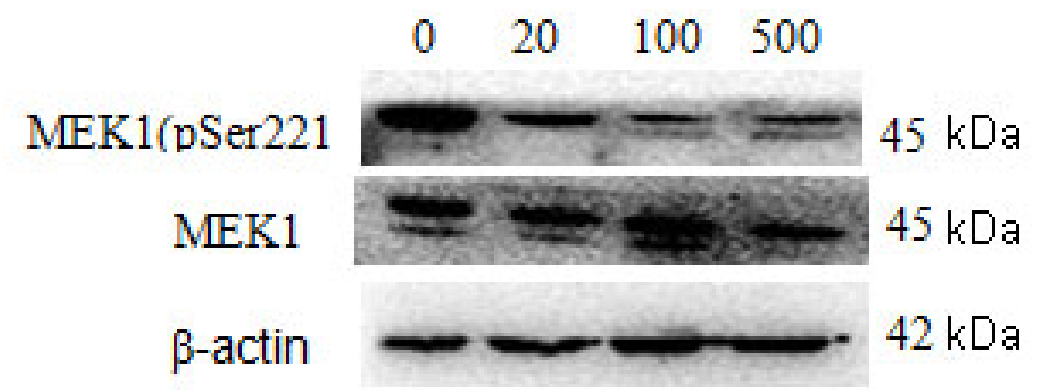

Figure 4. MAPK and p-MAPK expression of Jurkat T lymphocytes treated with different concentrations of acrylonitrile. 


\section{DISCUSSION}

The cell viability results showed that at higher concentrations of ACN, the OD value decreased, indicating a longer ACN processing period in cells and higher cytotoxicity. There was a statistically significant difference between the exposure group and control group $(\mathrm{P}<0.05)$.

Cholesterol modulates membrane fluidity over a range of physiological temperatures. Lipid rafts are dynamic cell-surface regions that are rich in cholesterol, saturated fatty acids, and sphingolipids. Within cells, cholesterol is a precursor molecule in several biochemical pathways, such as the synthesis of vitamin D and steroid hormones, including the adrenal gland hormones cortisol and aldosterone as well as the sex hormones progesterone, estrogens, testosterone, and their derivatives (Patel et al., 2008). Cholesterol and saturated fatty acid chains show high affinity and closely interact. When lipid rafts are destroyed, the membrane cholesterol content decreases; thus, cholesterol content can indirectly reflect the impact of ACN on lipid rafts (Ben-Dov and Korenstein, 2013).

Cholesterol is required to build and maintain cell membranes. Within the cell membrane, cholesterol also functions in intracellular transport, cell signaling, and nerve conduction. Cholesterol is essential for the structure and function of invaginated caveolae and clathrincoated pits, including caveola-dependent and clathrin-dependent endocytosis (Ramírez-Sánchez et al., 2012). Our study showed that when cells received ACN antigenic stimulation, lipid rafts showed dynamic changes and enriched signaling molecules to the aggregation site. ACN disrupted lipid rafts in a dose-dependent manner; at higher ACN concentration, cholesterol content was lower, causing greater degradation of lipid rafts. There were statistically significant differences between each contamination group and the control group $(\mathrm{P}<0.05)$.

$\mathrm{Bcl10}$ is a joint protein that can trigger intracellular signal transduction. After antigen stimulation, it will promote the secretion of interleukin-2, a molecule that plays an important role in the process of gene activation, lipid raft gathering, and $\mathrm{T}$ cell differentiation and maturation (Xu et al., 2009; Khalaf et al., 2010; Guo et al., 2012).

Caveolae are plasma membrane invaginations that are $60-80 \mathrm{~nm}$ in diameter and are subsets of lipid rafts enriched in cholesterol and sphingolipids; they contain at least 3 subunits, including caveolae-1, caveolae-2, and caveolae-3 (Fielding and Fielding, 2004; Feng et al., 2013). Caveolae are expressed in various tissues and cell types such as endothelial cells, macrophages, neutrophils, and adipocytes. The functions of caveolae are diverse and include endocytosis, transcytosis, potocytosis, calcium signaling, and the regulation of various signaling events. Pit is present in a variety of cell types in the special form of lipid rafts, most of which form the bottle type. Its invagination structure links different organelles with protein and regulates membrane communication. Caveolin- 1 is used as a lipid raft marker protein and allosteric proteins, which regulate communication between membranes and play an important role in signaling processes (Brennan et al., 2012; Boscher and Nabi, 2012). In the absence of caveolin-1, free cholesterol accumulates in mitochondrial membranes, increasing membrane condensation and reducing the efficiency of the respiratory chain and intrinsic antioxidant defense. Upon activation of oxidative phosphorylation, reactive oxygen species accumulation is promoted, resulting in cell death. It has been reported that this mitochondrial dysfunction predisposes caveolin1-deficient animals to mitochondrial-related diseases such as steatohepatitis and neurodegeneration (Bosch et al., 2011).

It was reported that $\mathrm{Bcl} 10$ is an apoptotic regulatory molecule that is closely associated with immune responses (Bhattacharyya et al., 2007). Bcl10 is a joint protein that is 
important in lymphocyte development and signaling pathways. Our results indicate that with increasing $\mathrm{ACN}$ concentration, total Bcl10 concentration remains constant, but is mainly present in the lower area. Our data suggest that ACN may cause immune toxicity by damaging lipid raft structures, resulting in Bcl10 protein and lipid raft separation.

It is thought that a variety of cell surface lipid rafts come together to form a contact point known as the immunological synapse. Therefore, lipid rafts are a necessary component of the immune synapse: cells receive antigen stimulation, the signal is enriched to stimulate parts of the molecular aggregates, and lipid rafts undergo dynamic changes. For example, the inner layer of plasma membrane contains acytelated tyrosine protein kinase family (Ishikawa et al., 2013). Lipid rafts are dispersed in the plasma membrane and can drift sideways. Therefore, lipid rafts can activate receptors, causing signal conversion elements to come together and gene regulation via protein kinase phosphorylation. Studies have shown that the same receptors in the plasma membrane lipid raft and non-raft areas may determine their distribution pattern and activate different downstream signaling pathways (Magee et al., 2002).

The Ras/Raf/MAPK/ERK pathway is an intracellular signaling cascade that is a very important transfer pathway. The pathway consists of a 3-enzyme functional unit; Raf, MAPK, and ERK are activated by phosphorylation (Robbs et al., 2013). Under physiological conditions, ERK is the only downstream substrate of MAPK. This indicates that the MAPK pathway plays an important role in signaling. The ERK pathway induces expression of caveolin-1, allowing the cell cycle to achieve G1/S phase transition, and is also closely related to cell proliferation and growth inhibition (Zhu et al., 2011; So and Croft, 2012). Studies have shown that MAPK plays an important role in maintaining ERK1/2 activation and cell cycle regulation. Therefore, we analyzed MAPK and p-MAPK levels to reflect protein redistribution after ACN exposure in the signaling pathway. We found that MAPK protein appeared as dark gray bands at 20 and $100 \mu \mathrm{M} \mathrm{ACN}$, and as lighter gray bands at $500 \mu \mathrm{M}$. p-MAPK concentration increased after ACN exposure, while MAPK protein secretion increased at specific ACN concentrations. Cell viability and MAPK protein secretion decreased beyond a specific ACN concentration. The grayscale of the p-MAPK band of contamination groups was lower than that of the control group with increasing ACN concentration, while p-MAPK content showed a decreasing trend. Therefore, by destroying the structure of lipid rafts and allosteric protein redistribution, ACN may suppress the Raf/Ras/MAPK/ERK signaling pathway, which is important for immunotoxicity pathogenesis (Efferth, 2012).

This study used cells only from in vitro culture. Signaling in vivo is more complex and cell fate is affected by many factors such as mutual cooperation, antagonism, checks, and balances, and thus more accurately reflect the regulation of cell-specific responses. Additional studies are necessary to determine how MAPK activates Raf and the structures of its 3 isomers, MAPK gene polymorphisms, MAPK enhancers or inhibitors, and their effects on the signal transduction pathway.

\section{Conflicts of interest}

The authors have no conflict of interest to declare.

\section{ACKNOWLEDGMENTS}

Research supported by the Science Foundation of Shanghai Jinshan District Health 
Bureau (\#JSKJ-KTQN-201201) and the Shanghai Municipal Health Bureau, China (\#2013114). The authors thank Dr. Bing Li for assisting with Western blot analysis and Dr. Jimin Shi for assisting with cell culture.

\section{REFERENCES}

Ben-Dov N and Korenstein R (2013). Proton-induced endocytosis is dependent on cell membrane fluidity, lipid-phase order and the membrane resting potential. Biochim. Biophys. Acta 1828: 2672-2681.

Bhattacharyya S, Borthakur A, Pant N, Dudeja PK, et al. (2007). Bcl10 mediates LPS-induced activation of NF- $\mathrm{kB}$ and IL-8 in human intestinal epithelial cells. Am. J. Physiol. Gastrointest. Liver Physiol. 293: G429-G437.

Bosch M, Marí M, Herms A, Fernandez A, et al. (2011). Caveolin-1 deficiency causes cholesterol-dependent mitochondrial dysfunction and apoptotic susceptibility. Curr. Biol. 21: 681-686.

Boscher C and Nabi IR (2012). Caveolin-1: role in cell signaling. Adv. Exp. Med. Biol. 729: 29-50.

Brennan D, Peltonen S, Dowling A, Medhat W, et al. (2012). A role for caveolin-1 in desmoglein binding and desmosome dynamics. Oncogene 31: 1636-1648.

Efferth T (2012). Signal transduction pathways of the epidermal growth factor receptor in colorectal cancer and their inhibition by small molecules. Curr. Med. Chem. 19: 5735-5744.

Feng H, Guo W, Han J and Li XA (2013). Role of caveolin-1 and caveolae signaling in endotoxemia and sepsis. Life Sci. 93: 1-6.

Fielding CJ and Fielding PE (2004). Membrane cholesterol and the regulation of signal transduction. Biochem. Soc. Trans. 32: 65-69.

Guo CJ, Wu YY, Yang LS, Yang XB, et al. (2012). Infectious spleen and kidney necrosis virus (a fish iridovirus) enters Mandarin fish fry cells via caveola-dependent endocytosis. J. Virol. 86: 2621-2631.

Hancock JF (2006). Lipid rafts: contentious only from simplistic standpoints. Nat. Rev. Mol. Cell Biol. 7: 456-462.

Huang J, Ma GJ, Sun NN, Wu ZF, et al. (2010). BCL10 as a new candidate gene for immune response in pigs: cloning, expression and association analysis. Int. J. Immunogenet. 37: 103-110.

Ishikawa T, Yuhanna IS, Umetani J, Lee WR, et al. (2013). LXRß/estrogen receptor-alpha signaling in lipid rafts preserves endothelial integrity. J. Clin. Invest. 123: 3488-3497.

Khalaf H, Jass J and Olsson PE (2010). Differential cytokine regulation by NF- $\mathrm{kB}$ and AP-1 in Jurkat T-cells. BMC Immunol. 11: 26.

Kusumi A, Ike H, Nakada C, Murase K, et al. (2005). Single-molecule tracking of membrane molecules: plasma membrane compartmentalization and dynamic assembly of raft-philic signaling molecules. Semin. Immunol. 17: 3-21.

Magee T, Pirinen N, Adler J, Pagakis SN, et al. (2002). Lipid rafts: cell surface platforms for T cell signaling. Biol. Res. 35: 127-131.

Patel HH, Murray F and Insel PA (2008). Caveolae as organizers of pharmacologically relevant signal transduction molecules. Annu. Rev. Pharmacol. Toxicol. 48: 359-391.

Pike LJ (2006). Rafts defined: a report on the keystone symposium on lipid rafts and cell function. J. Lipid Res. 47: 1597 1598.

Ramírez-Sánchez I, Mendoza-Lorenzo P, Zentella-Dehesa A, Méndez-Bolaina E, et al. (2012). Caveolae and non-caveolae lipid raft microdomains of human umbilical vein endothelial cells contain utrophin-associated protein complexes. Biochimie 94: 1884-1890.

Robbs BK, Lucena PI and Viola JP (2013). The transcription factor NFAT1 induces apoptosis through cooperation with Ras/Raf/MEK/ERK pathway and upregulation of TNF- $\alpha$ expression. Biochim. Biophys. Acta 1833: 2016-2028.

Simons K and Vaz WL (2004). Model systems, lipid rafts, and cell membranes. Annu. Rev. Biophys. Biomol. Struct. 33: 269-295.

Simons K and Gerl MJ (2010). Revitalizing membrane rafts: new tools and insights. Nat. Rev. Mol. Cell Biol. 11: 688-699.

So $\mathrm{T}$ and Croft M (2012). Regulation of the PKCtheta-NF- $\mathrm{kB}$ Axis in T lymphocytes by the tumor necrosis factor receptor family member OX40. Front Immunol. 3: 133.

Szoor A, Szollosi J and Vereb G (2010). Rafts and the battleships of defense: the multifaceted microdomains for positive and negative signals in immune cells. Immunol. Lett. 130: 2-12.

Xu S, Huo J, Lee KG, Kurosaki T, et al. (2009). Phospholipase Cgamma2 is critical for Dectin-1-mediated $\mathrm{Ca}^{2+}$ flux and cytokine production in dendritic cells. J. Biol. Chem. 284: 7038-7046.

Yang CH, Tian L, Ling GS, Trendell-Smith NJ, et al. (2008). Immunological mechanisms and clinical implications of regulatory $\mathrm{T}$ cell deficiency in a systemic autoimmune disorder: roles of IL-2 versus IL-15. Eur. J. Immunol. 38 : $1664-1676$. 
Young RM, Holowka D and Baird B (2003). A lipid raft environment enhances Lyn kinase activity by protecting the active site tyrosine from dephosphorylation. J. Biol. Chem. 278: 20746-20752.

Yuanqing H, Suhua W, Guangwei X, Chunlan R, et al. (2013). Acrylonitrile has distinct hormetic effects on acetylcholinesterase activity in mouse brain and blood that are modulated by ethanol. Dose Response 11: 49-59.

Zhu X, Zhou F, Qin D, Zeng Y, et al. (2011). Human immunodeficiency virus type 1 induces lytic cycle replication of Kaposi's-sarcoma-associated herpes virus: role of Ras/c-Raf/MEK1/2, PI3K/AKT, and NF-кB signaling pathways. J. Mol. Biol. 410: 1035-1051. 